\title{
Liderazgo Escolar en Tiempos de Crisis. El Caso de dos Liceos del Centro Sur de Chile después del 27F
}

\author{
School Leadership during a Crisis. The Case of two Chilean \\ High Schools after 27F
}

\author{
Javier Pascual * \\ Denis Larraguibel \\ Daniela Zenteno \\ Francisca Guarda
}

Centro de Innovación en Educación - Fundación Chile

\begin{abstract}
El presente artículo tiene como objetivo identificar prácticas de liderazgo y gestión escolar efectivas en tiempos de crisis dentro de los establecimientos educativos, que permitan mantener un funcionamiento normal durante el tiempo que lleve su resolución. Se entiende como crisis un cambio de mayor intensidad que produce un desajuste en el funcionamiento de una organización, y entre ésta y el contexto. Para cumplir con el objetivo de investigación, se realizó un estudio de casos en el que se hizo un seguimiento de dos liceos de la zona centro sur de Chile que fueron damnificados por el terremoto ocurrido en febrero de 2010, a través de metodologías mixtas que incluyeron entrevistas, focus groups, encuestas, técnicas proyectivas y revisión de información secundaria. Se identificaron tres líneas de acción relevantes: a) mantener el foco en lo pedagógico a pesar de las adversidades; b) propiciar un liderazgo distribuido, empoderando a los actores relevantes y promoviendo la participación escolar; y c) asegurar una estrategia de comunicación que sea para todos, permanente y por canales formales para reducir los niveles de ansiedad. Adicionalmente, cuando es pertinente, se vuelve importante la apertura hacia el apoyo externo y la asesoría sin miedo a mostrar las debilidades internas.
\end{abstract}

Descriptores: Liderazgo escolar, Crisis, Liderazgo distribuido, Participación escolar, Comunicación interna.

\begin{abstract}
This paper aims to identify effective school leadership and management practices during a crisis period. We define crisis as a change of great intensity which produces a school operation imbalance within the organization and with its surrounding environment. We are particularly concerned about those practices that allow coping with the context of adversity and keeping normal operation. In order to identify those practices, we conducted a case study of two high schools that were highly affected by the Chilean massive earthquake on February 2010, the sixth largest in the world ever recorded. These schools were monitored for four years using mixed methods, including interviews, focus groups, surveys, projective techniques and secondary information review. As a result, we identify three main effective strategies: a) ensuring a focus on pedagogy despite all the other concerns; b) empowering others to distribute leadership and spread the school community support, promoting wider participation of the entire school community including parents and students; and c) ensuring permanent formal communication to the entire community in order to reduce anxiety and avoid misunderstandings. Additionally, when available, it is important to be open for external support despite the risk of showing internal weaknesses.
\end{abstract}

Keywords: School leadership, Crisis, Distributed leadership, Participation, Internal communication.

*Contacto: jpascualmedina@gmail.com

ISSN: $1696-4713$

www.rinace.net/reice/
Recibido: $\quad 8$ de febrero 2015

$1^{\text {a }}$ Evaluación: 28 de abril 2015

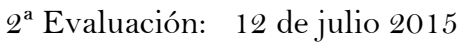

Aceptado: 22 de agosto 2015 


\section{Introducción}

El 27 de febrero de 2010, un terremoto de mayor intensidad sacudió la zona centro sur de Chile. Se cuantificaron después de la catástrofe 130 establecimientos educacionales afectados con daños totales o de reposición en más de un $50 \%$ de su infraestructura, de los cuales el $90 \%$ eran establecimientos municipales de grupo socioeconómico bajo y medio-bajo (Mineduc, 2013). Si bien los establecimientos comenzaron a funcionar 45 días después de ocurrido el terremoto, en varios casos tuvieron que trasladarse a escuelas modulares, o funcionar en condiciones más limitadas.

Elacqua, Santos y Salazar (2010) señalan que cuando ocurre una crisis que afecta el funcionamiento de un establecimiento educacional, es primordial no sólo reestablecer las condiciones físicas del establecimiento, sino también estar focalizado en volver a las prácticas pedagógicas y a las metas de mejora propuestas lo antes posible. Las consecuencias de un terremoto pueden repercutir durante un tiempo extenso, y durante este período, los establecimientos deben continuar sus funciones sin perder de foco su misión pedagógica. En tiempos de crisis, esta tarea se vuelve un verdadero desafío, no sólo por las precarias condiciones a las que probablemente se enfrenten, sino además por los numerosos factores relacionados a la resolución de la crisis que pueden distraer a los directivos del establecimiento de la misión de la institución.

El terremoto de 2010 no sería la primera ni la última crisis que afectaría a nuestras escuelas. Sólo durante el año 2014, más de 30 establecimientos en la zona norte presentaron daños estructurales por el terremoto ocurrido en abril, mientras que dos escuelas se vieron damnificadas por un gran incendio en Valparaíso. En 2015 una gran inundación afectó la zona norte del país, y recientemente, en septiembre, otro terremoto y tsunami afectó la zona centro-norte. Por lo mismo, vale la pena preguntarse de qué manera los líderes educativos deben reaccionar durante tiempos en que su escuela no puede desarrollar sus funciones con normalidad, y cómo deben enfrentarlas sin perder de vista el foco pedagógico que debe primar en cualquier establecimiento educativo. El objetivo de este estudio, por lo tanto, es identificar elementos importantes para el liderazgo y la gestión en tiempos de crisis dentro de los establecimientos educativos, que permitan sobrellevar las adversidades y mantener un funcionamiento normal durante el tiempo que lleve su resolución.

\section{Revisión de la Literatura}

Uno de las primeras aclaraciones conceptuales a tener presente es el concepto de crisis. Dada la escaza literatura sobre crisis en instituciones escolares, la conceptualización que se utilizará hace referencia al común de las organizaciones, cualquiera sea ésta. Dora Fried Schnitman (2011) define como crisis a aquellos "cambios de diversa intensidad, bruscos o crónicos, que producen un desajuste en el funcionamiento de una organización, y entre ésta y el contexto" (p. 12). Para Fried Schnitman una crisis es un momento complejo y doloroso que puede producir más disenso que consenso entre las personas involucradas, y se encontraría ligada directamente con el afrontamiento, es decir, con el hecho de hacerle frente a dicha crisis llegando a entenderla como una oportunidad de cambio y construcción de un nuevo futuro. Las crisis serían, por lo tanto, instancias que generan desconcierto, estrés y plantean un desafío, pudiendo impactar en el sentido de identidad y la validez de las relaciones, provocando la necesidad de generar nuevos 
sentidos y nuevas relaciones. Sin embargo, éstas serían endémicas a las organizaciones, por lo que ponen a prueba las habilidades y capacidades para afrontar desafíos y organizarse de modo de lograr sobreponerse y avanzar más allá de donde los encontró la crisis (Fried Schnitman, 2011).

Cualquier crisis requiere que la persona al mando ejerza un liderazgo sólido. De acuerdo con Robinson, Hohepa y Lloyd (2009), el liderazgo educativo se caracteriza por la capacidad de influir en otros para que realicen acciones que se espera que mejoren los resultados educativos. Es por ello que Marfán y Horn (2012) señalan que éste puede ser entendido como "influencia". Reeves (2012) señala que un liderazgo bien ejercido es integral, e impacta a todas las variables relacionadas a resultados, preocupándose de tener un proyecto educativo común y conocido por toda la comunidad educativa que permita clarificar los objetivos y dar un sentido al trabajo realizado, manteniendo una buena comunicación entre directivos y docentes, e involucrando más a los apoderados en el establecimiento. El autor señala entre las prácticas de liderazgo directivo que afectan el clima escolar, el preocuparse de que el trabajo para la comunidad educativa sea satisfactorio -de modo que sientan un real compromiso con él-, valorar y reconocer el desempeño de cada actor, ser un real apoyo para éstos, generar altas expectativas de rendimiento en alumnos y docentes y dar a conocer los logros a la comunidad. El liderazgo se constituye como un factor fundamental para obtener buenas condiciones materiales, cognitivas y psicológicas que permitan un trabajo pedagógico eficaz en la escuela, ejerciendo acción directa sobre las variables mediadoras como la motivación docente y las condiciones de la escuela, e indirecta sobre el aprendizaje de los alumnos (Valenzuela y Horn, 2012).

Dentro de los distintos estilos de liderazgo existente, destaca por su eficacia el liderazgo distribuido (Harris, 2012). Este permite que las responsabilidades de gestión y administración no recaigan completamente sobre el director, sino también sobre los docentes, incorporando así un gran aliado. Según Harris, el liderazgo distribuido tiene tres implicancias: supone una distribución de poder menos vertical, donde las jerarquías son más difusas; implica una distribución de tareas al interior de la escuela distinta a las tradicionales; e introduce la posibilidad de que todos los docentes se vuelvan líderes en diferentes momentos. El liderazgo distribuido entrega la posibilidad a los profesores de liderar, lo que tendría una influencia positiva tanto en las relaciones sociales dentro de la escuela como en el desempeño de sus labores, pues se potencia que ellos se apropien de las prácticas de mejora, a la vez que permite que el director pueda ocupar su tiempo en otras tareas. Pero el liderazgo distribuido no se queda sólo ahí, no implica sólo compartir el liderazgo con los docentes, sino que refiere a un trabajo con toda la comunidad educativa, incorporando también a asistentes de la educación, apoderados y estudiantes en la toma de decisiones, logrando un trabajo conjunto que les permita afrontar diversas dificultades y desarrollar tareas, retos o metas propuestas (Harris, 2002). De este modo, la práctica del liderazgo se realiza a través de la participación, interacción y acción de todos los actores educacionales involucrados. Se entiende entonces el liderazgo distribuido como el resultado de actividades concertadas y conjuntas de un grupo red, donde todos los actores son agentes de cambio, y ostentan un rol de liderazgo en cada trasformación (García, 2010; Murillo, 2006).

Existen varias medidas que utilizan los directores para afrontar dificultades dentro del establecimiento. Leithwood y Stager (2009) concluyeron que los directores expertos enfrentan un problema no estructurado, como una crisis, reflexionando primeramente 
sobre el problema y las consecuencias que tendrá para el funcionamiento de la escuela y para el aprendizaje de los alumnos, logrando una visión más global y clara de su impacto en la comunidad, generando a partir de esto una planificación y estrategia detallada para poder sobrellevar el problema.

Un foco muy importante frente a una crisis organizacional es la contención emocional y la reducción de los niveles de estrés que se pueden generar entre los actores. El informe anual sobre Derechos Humanos de 2010 de la Universidad Diego Portales señala que dado el terremoto ocurrido en Chile el 27 de febrero de ese año, muchos niños y jóvenes sufrieron estrés postraumático, con sensaciones de inseguridad y miedo, volviéndose más dependientes, sobre todo si lugares significativos para ellos, como plazas o colegios, sufrieron daños o quedaron inutilizables. En este mismo informe se menciona que en la Convención sobre los Derechos del Niño (CDN) se establece que los Estados deben "recuperar física y psicológicamente a los niños afectados por una situación de desastre" (Universidad Diego Portales, 2010, p. 318), aunque de lo observado en el estudio se deduce que en Chile muchas veces las fuerzas se concentran más en mejorar los bienes materiales que en dar apoyo emocional. El Instituto Nacional de Derechos Humanos indica que cambios de ambientes (como escuelas o domicilios), sumado a las alteraciones emocionales de los estudiantes y docentes, generan un ambiente escolar más tenso y conflictivo (INDH, 2012). Leithwood (2009) asegura que cómo se sientan y cómo piensen los docentes afecta su desempeño pedagógico, de tal forma que sus estados emocionales, como el estrés, el ánimo, o el compromiso organizacional, pueden influenciar fuertemente su trabajo. El autor indica que una de las medidas que más influye en las emociones de los profesores son las condiciones laborales en que se desenvuelven, sobre todo la carga de trabajo, teniendo también una fuerte influencia el apoyo que percibe por parte del equipo directivo. Esto deja ver que es fundamental velar por el estado anímico de los docentes -tarea en la cual los líderes educativos tienen un importante papel según comenta Leithwood-, ya que en una situación de crisis que lleva a empeorar las condiciones laborales y a aumentar la carga de trabajo podría generar estrés en los profesores.

Una crisis puede producir diversas dificultades dentro de una comunidad. Transberg (2012) indica como una de las medidas importantes para mitigar las dificultades que se producen durante un proceso de reconstrucción -basándose en el caso de Haití tras el terremoto de 2010- es la transparencia de información, es decir, ser lo más claro y sincero posible con los distintos actores, entregando toda la información acerca de los pasos a seguir y sus respectivos plazos, así como sobre la rendición de cuentas, manteniendo a la comunidad continuamente actualizada sobre los avances y los problemas surgidos, de forma de evitar cualquier tipo de conflicto o malentendido, ya que con la trasparencia de información se da una mayor seguridad a los involucrados, quienes son finalmente los principales afectados e interesados, posibilitando así una mayor eficacia en la resolución del problema. Especialmente en escuelas que cuentan con asesoría técnica externa para afrontar la crisis -medida que ha tomado fuerza en Chile en el último tiempo efecto de la Ley de Subvención Escolar Preferencial (Ley SEP)- se hace relevante que exista transparencia de información y que se genere confianza entre la institución asesora y el establecimiento en cuestión, de tal modo que los esfuerzos se basen en datos reales y se logre una ayuda efectiva a la escuela. Sotomayor y Sánchez (2010), señalan que uno de los aspectos que puede generar resistencia por parte de una escuela a una asesoría externa es la desinformación que puedan sentir. Sin embargo, 
estas resistencias se logran superar a medida que avanza la asesoría, mediante algunas acciones que asegurar una mejor relación entre la escuela y la institución asesora (Bellei, Raczynski y Osses, 2010). Estas medidas permitirían un trabajo más articulado entre ambas instituciones, formando un vínculo fuerte, de confianza y apoyo, que logre difuminar la resistencia inicial, y resolver de mejor forma las problemáticas de la escuela (Sotomayor y Sánchez, 2010).

En resumen, la literatura sugiere tres líneas para el afrontamiento de una crisis. Un liderazgo sólido y preferentemente distribuido, la contención emocional de toda la comunidad escolar y una comunicación interna fluida y transparente, y todas estas líneas de acción tendrían como meta el funcionamiento normal, o lo más cercano posible a la normalidad, del establecimiento durante la crisis.

\section{Metodología}

El presente artículo es un estudio de tipo exploratorio, pues intenta dar a conocer elementos clave dentro de las múltiples prácticas de gestión y liderazgo de los equipos directivos que permitan sobrellevar una crisis. Para esto, se ha realizado un estudio de casos, con un seguimiento en profundidad a dos liceos de la zona centro-sur de Chile que fueron devastados por el terremoto ocurrido el 27 de febrero de 2010. Estos establecimientos son el Liceo Claudina Urrutia de Lavín (LCU) y el Liceo Polivalente Mariano Latorre (LML), ambos de enseñanza media. A ambos liceos se les hizo un seguimiento longitudinal, dentro del contexto de una consultoría institucional y acompañamiento durante el proceso proporcionado por el Centro de Innovación en Educación de Fundación Chile, por lo que el estudio se acerca a los lineamientos de una investigación-acción. Este seguimiento observó a todos los integrantes de la comunidad escolar durante distintas instancias, las cuales se describen a continuación:

- Diagnóstico base y de expectativas: A través de un trabajo cualitativo que incluyó entrevistas a directivos, docentes y asistentes de la educación, y focus groups con alumnos y con apoderados, se recogieron y analizaron las expectativas de los actores sobre la reconstrucción, a la vez que se conocieron las dinámicas regulares de los liceos anteriores a la crisis. Este diagnóstico se realizó a fines del año 2010, y concluyó con un informe por escuela dirigido tanto a los directivos como a los encargados de la reconstrucción y al equipo de asesoría externa.

- Seguimiento y monitoreo: Los liceos fueron visitados en cinco oportunidades entre los años 2011 y 2012, con el objetivo de monitorear su funcionamiento, clima y principales problemáticas durante el proceso de reconstrucción. Para esto se hizo trabajo cuantitativo y cualitativo, a través de encuestas a todos los docentes y asistentes de la educación y una muestra representativa de alumnos -que según el periodo, la representatividad se tradujo en errores de estimación máximos de entre $4,2 \%$ y $5,2 \%$ a un $95 \%$ de confianza-, y focus groups con los equipos directivos. En una de estas visitas se hizo un trabajo plenamente cualitativo, con entrevistas y focus groups, donde participaron representantes de los mismos actores mencionados. En todos los instrumentos se abordaron temáticas sobre flujos de información y comunicación dentro del establecimiento, clima y convivencia escolar, prácticas de liderazgo directivo. 
También se incluían algunos temas emergentes según la contingencia local y nacional, en una sección más variable que ayudaba a comprender los cambios en las respuestas de los temas longitudinales. De cada monitoreo surgía un informe de resultados por cada establecimiento, dirigido tanto a los correspondientes equipos directivos como al equipo de asesoría externa, con el objetivo de tomar acciones remediales.

- Evaluación final y taller retrospectivo: Ya finalizado cada proceso de reconstrucción, se realizó un taller con representantes de directivos, docentes, asistentes de la educación, alumnos y apoderados, cuyo objetivo fue reflexionar sobre el proceso vivido en tiempos de crisis. Para ello, se recordaron los principales hitos entre el terremoto de 2010 y el momento del taller, los cuales se representaron de manera gráfica para luego estudiarlos a través de un análisis proyectivo. De estos talleres surgió un informe para cada liceo y para el equipo de asesoría externa.

El presente artículo corresponde a una metasíntesis de los informes generados durante los cuatro años de seguimiento, los cuales cuentan con información tanto cuantitativa como cualitativa. Para complementar la información con datos más objetivos, se realizaron algunos análisis de bases de datos públicas como lo son los datos de matrícula o los resultados SIMCE. Además se realizó una revisión de los documentos de gestión que se elaboraron durante todo el proceso de reconstrucción, los cuales registran los avances y resoluciones de las mesas técnicas que articularon dicho proceso.

La información de las distintas instancias fue sintetizada en función de los objetivos de este estudio para la extracción de los principales resultados, los cuales se centran no en el proceso de reconstrucción, sino en la gestión y las prácticas de liderazgo que los directores de ambos establecimientos asumen durante los cuatro años en situación de crisis que vivieron los liceos. Cabe destacar que, como metasíntesis cualitativa, la presentación posterior de los resultados no se realizará instrumento por instrumento, dadas las limitaciones de espacio, sino que se presentarán los principales aprendizajes basados en la triangulación de los datos obtenidos en los distintos informes e instrumentos, y en función de los objetivos del estudio y las líneas propuestas por la revisión bibliográfica, los cuales se reforzarán con los ejemplos de resultados primarios como gráficos o citas de entrevistas extraídos directamente de los informes.

Es importante tener en cuenta que, como cualquier estudio de casos exploratorio, este estudio no pretende de ninguna manera otorgar resultados generalizables para todos los establecimientos escolares, pues no es posible en base a sólo dos casos específicos. El objetivo central que atiende esta metodología es de identificar las principales líneas en las que se enmarcan las prácticas que en estos establecimientos hayan sido exitosas, con el fin de dar pistas a otros y guiar futuras investigaciones. Se debe recordar que cada contexto escolar es único, y no siempre las mismas prácticas son efectivas, pero sí se puede dar luces de qué líneas seguir y qué elementos considerar al momento de actuar frente a una crisis. 


\section{Antecedentes de los Casos}

Antes de pasar a los resultados, resulta importante conocer la historia y características de los liceos, de manera de poder darle contexto a las prácticas de liderazgo que fueron observadas.

Ambos liceos son municipales, pertenecen a la Red de Escuelas Líderes de Educación contra la pobreza y han formado parte de programas de mejoramiento educativo-MECE media- e innovación pedagógica -Programa Montegrande- impulsados por el Ministerio de Educación, mostrando altas capacidades de gestión y liderazgo, constituyéndose en referentes al interior de sus comunidades locales. Si bien comparten todo esto, cada uno tiene sus propias particularidades, las cuales se describen a continuación.

\subsection{Liceo Claudina Urrutia de Lavín, Cauquenes}

El Liceo Claudina Urrutia de Lavín, fue inaugurado en 1901 como el Liceo de Niñas de Cauquenes (hoy es mixto), teniendo al momento del terremoto casi 110 años de historia. Está ubicado en la plaza principal de Cauquenes, e imparte educación de $1^{\circ}$ a $4^{\circ}$ medio, en modalidad científico humanista. Su matrícula actual es cercana a los 900 estudiantes, y concentra altos índices de vulnerabilidad socioeducativa. Se ha destacado por la generación e implementación de proyectos de innovación pedagógica orientados a fortalecer las competencias, habilidades y actitudes de sus estudiantes para asegurarles mejores trayectorias post secundarias, sean éstas continuidad de estudios o inserción al mundo laboral. Entre estos proyectos se encuentran los planes de Orientación para el trabajo en $3^{\circ}$ y $4^{\circ}$ medio; fortalecimiento de habilidades del pensamiento en $1^{\circ}$ y $2^{\circ}$ medio; planes de fortalecimiento de la comprensión lectora como habilidad transversal al currículum en $1^{\circ}$ y $2^{\circ}$ medio y pasantías nacionales e intercambio de experiencias con otros establecimientos.

Sus resultados de aprendizaje en lenguaje y matemática medidos por el SIMCE en $2^{\circ}$ medio, destacan al compararlos con establecimientos similares, situándose sobre la media nacional. En tanto, los promedios PSU de los últimos tres años se encuentran entre los 465 y 525 puntos aproximadamente, rindiendo la prueba un porcentaje cercano al $90 \%$ de la matrícula de $4^{\circ}$ medio, distinguiendo un número menor de estudiantes que obtiene puntajes sobresalientes.

Este Liceo es liderado por un director con 7 años en el cargo, pero que previamente se desempeñó en distintos roles y tareas dentro del liceo, lo que le permite tener una visión muy completa del quehacer de la institución. Comparte su trabajo con un equipo de gestión de tres personas (Inspectora, Jefa de UTP y Orientadora) y con alrededor de 35 profesores, algunos de los cuales contribuyen a potenciar el trabajo de la unidad técnico pedagógica como encargados de subsectores.

Aunque este liceo comparte la tarea de educación media con otros liceos de la comuna, se destaca por su apertura a la comunidad, contando con redes de colaboración conformada por distintas instituciones públicas y privadas y desarrollando actividades para los jóvenes de la comuna como son ferias vocacionales, actividades culturales y deportivas.

El terremoto de 2010 causó serios daños estructurales en el establecimiento que provocaron que fuese muy riesgoso el ingreso, por lo que se debieron iniciar las actividades dos meses más tarde en unas dependencias facilitadas por el DAEM de 
Cauquenes, donde se instalaron aulas modulares y se aprovecharon algunas aulas ya existentes. En los primeros meses fueron notorias algunas necesidades como de servicios higiénicos y otros espacios, las cuales fueron atendidas, aunque el proceso duró tres años y medio, y en todo ese tiempo el liceo continuó sus funciones en condiciones provisorias. Durante 2011 se tomó la decisión de demoler el liceo por sus daños estructurales, y no fue hasta 2012 que la obra comenzó a ser visible. Si bien el proyecto contemplaba el inicio de actividades escolares en el nuevo liceo a principios de 2013, la obra se retrasó, lo que obligó a efectuar la mudanza en vacaciones de invierno, lo que conllevó a que se inaugurara para el aniversario 110 de la institución, a inicios del segundo semestre del mismo año.

\subsection{Liceo Polivalente Mariano Latorre, Curanilahue}

El Liceo Mariano Latorre, fue inaugurado en 1964, y al momento del terremoto tenía 46 años de trayectoria como único liceo municipal de Curanilahue, Su matrícula asciende a 1.300 estudiantes aproximadamente y su índice de vulnerabilidad educativa es de $60 \%$. Imparte educación de $1^{\circ}$ a $4^{\circ}$ medio en modalidad Polivalente, es decir, CientíficoHumanista (HC) y Técnico Profesional (TP), actualmente con las especialidades de electrónica y mecánica industrial, aunque también con especialidad en madera hasta antes del terremoto. Cuenta además con una oferta importante en el área Artística, talleres de música, danza, artes visuales y teatro, la cual se combina con las anteriores (TP y HC).

El liceo Mariano Latorre desarrolla desde el año 1998 el proyecto de la Orquesta Sinfónica, que posteriormente derivó en la Orquesta Infantil y Juvenil del Bicentenario, proyecto que ha hecho posible potenciar los talentos artísticos de los niños, niñas y jóvenes de la comuna. Su trayectoria ha estado marcada por la innovación y deseo de ampliar las oportunidades de sus estudiantes. Tal es el caso de la creación de planes y programas propios de la modalidad de educación artística en $3^{\circ}$ y $4^{\circ}$ medio; el desarrollo de programas de colaboración con Universidades, expandiendo las alternativas post secundarias y continuidad de estudios de los jóvenes; el programa integral de nivelación de habilidades básicas en $1^{\circ}$ medio; el desarrollo y ejecución del Proyecto de Radio Escolar; los planes de fortalecimiento de la comprensión lectora como habilidad transversal en $1^{\circ}$ y $2^{\circ}$ medio; pasantías nacionales e Internacionales y la participación en seminarios de intercambio de experiencias de innovación.

Sus resultados de aprendizaje en lenguaje y matemática medidos por el SIMCE en $2^{\circ}$ medio, destacan al compararlos con sus iguales según nivel socioeconómico, situándose sobre la media nacional. En relación a los puntajes de la PSU, los promedios de los últimos tres años se encuentran entre 474 y 531 puntos, rindiendo la prueba el $77 \%$ de la matrícula del $4^{\circ}$ medio, distinguiendo un número menor de estudiantes que obtiene puntajes sobresalientes.

Este Liceo es liderado por un director subrogante, con 6 años de ejercicio en el cargo, pero que previamente se desempeñó en distintos roles y tareas en el liceo, que le permiten tener una visión muy completa del quehacer de la institución. Comparte su trabajo dentro de un equipo amplio de 14 personas, entre las que hay docentes y asistentes de la educación, que representan distintas áreas del Liceo como Inspectoría, UTP y Currículum, Artística, Técnico Profesional, Orientación, Servicios Juveniles y Finanzas. Por su parte, la Unidad Técnico Pedagógica que lidera el trabajo de más de 80 profesores, distribuye sus tareas en un equipo de personas que lidera distintos procesos 
claves para la enseñanza desde la coordinación de niveles y asignaturas, currículum y evaluación.

Este Liceo, como único establecimiento municipal, se destaca por su articulación con otros, ya sean organizaciones internacionales y empresas que apoyan el desarrollo de la $\mathrm{TP}$, instituciones públicas que conforman una red de atención para los estudiantes, participación en red de establecimientos de educación básica municipal de la comuna y en red de Liceos Técnico Profesional de la provincia, entre otras.

Luego del terremoto de 2010, el liceo vio dañados dos tercios de su infraestructura, por lo que se decidió seguir funcionando dentro de las mismas dependencias, aunque debieron dividir a los alumnos en jornadas de mañana y tarde, haciendo clases a $3^{\circ}$ y $4^{\circ}$ medio en las mañanas y $1^{\circ}$ y $2^{\circ}$ medio en las tardes, y reducir las horas pedagógicas de 45 a 35 minutos. El año 2013 comenzó la reconstrucción física del liceo, lo que obligó el traslado a dos edificios escolares facilitados por el DAEM de Curanilahue, los cuales se encontraban a unos 20 minutos de viaje caminando. Las limitaciones de espacios de ambos edificios sólo permitían atender a una generación a la vez, lo que obligó a mantener la doble jornada e implicó para muchos de los docentes una carga mayor asociada a los traslados de una sede a otra durante su jornada. La obra se retrasó un año completo debido a problemas con la constructora, la cual fue reemplazada por otra en la mitad del proceso, por lo que el liceo comenzó su funcionamiento normal en sus nuevas dependencias a inicios de 2014, cumpliéndose cuatro años en condiciones provisorias.

\section{Resultados}

Lo primero que cabe destacar es que ambos establecimientos mantuvieron sus resultados académicos - medidos a través del Sistema de Medición de la Calidad de la Educación (SIMCE), prueba estandarizada bianual en $2^{\text {a }}$ medio- a pesar de las adversidades, tal como muestran los gráficos 1 y 2 -incluso el Liceo Mariano Latorre mejoró sus resultados en matemática, y tuvo una tendencia al alza en lenguaje- que se rompió sólo en 2013-. Si bien sus matrículas decrecieron, un análisis relativo a las matrículas comunales indica que las fluctuaciones respondieron más bien a movimientos demográficos de las ciudades en las que se ubican.

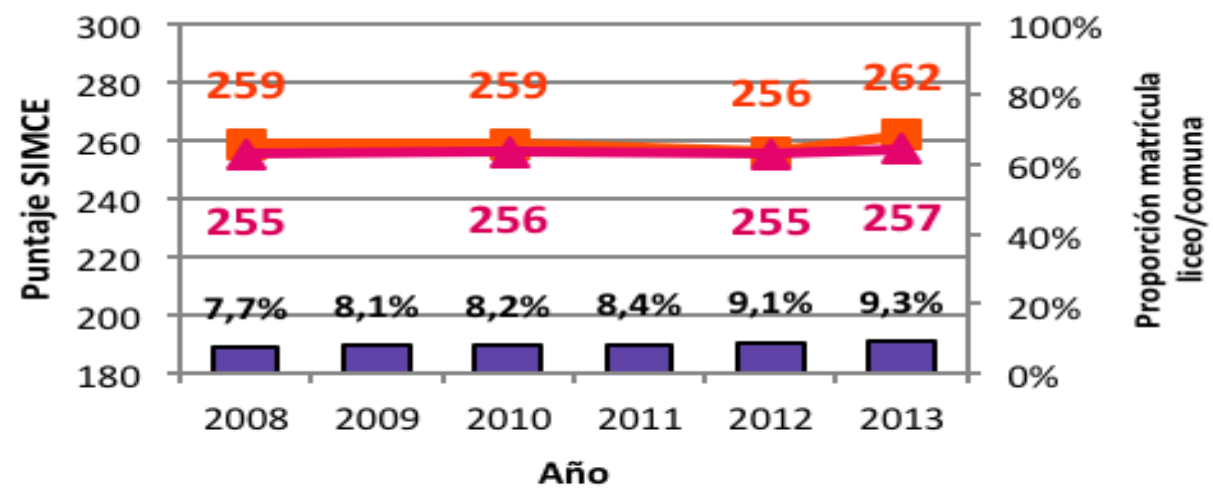

$\square$ Relación Matrícula SIMCE Lenguaje SIMCE Matemática

Gráfico 1. Tendencias de Matrícula y Resultados (LCU)

Fuente: Elaboración propia a partir de datos extraídos de bases SIMCE $2^{\circ}$ Medio y matrícula entre 2008 y 2013, MINEDUC. 
Se debe considerar que ambos equipos directivos debieron liderar el proceso de reconstrucción, administrar las múltiples demandas y responder adecuadamente a los requerimientos externos e internos que significó dicho proceso. Entre los nuevos aspectos a atender, se encuentra el fortalecimiento de la capacidad de gestionar la relación con el contexto, tanto referido a las autoridades y organismos públicos -como municipios, Departamentos de Administración de Educación Municipal (DAEM), Departamento de obras, Ministerio de Educación en sus distintos niveles (nacional, regional y provincial) y otras reparticiones públicas- como también con actores privados -la empresa financista, las oficinas de arquitectos, las empresas constructoras y la asistencia técnica externa-, quienes durante el periodo de reconstrucción, desarrollaron roles claves en la ejecución del proyecto.

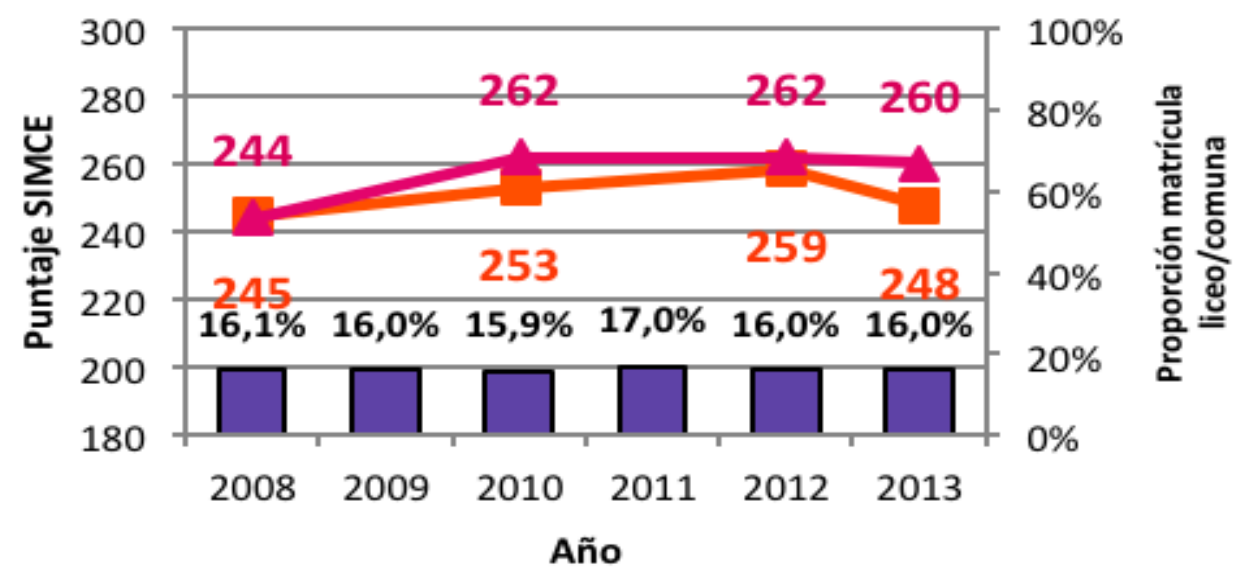

\footnotetext{
$\square$ Relación matrícula $\longrightarrow$ SIMCE Lenguaje $\longrightarrow$ SIMCE Matemática

Gráfico 2. Tendencias de matrícula y reusltados (LML)

Fuente: Elaboración propia a partir de datos extraídos de bases SIMCE $2^{\circ}$ Medio y matrícula entre 2008 y 2013, MINEDUC.
}

Para ambos equipos directivos, los requerimientos fueron diversos y muchos de ellos, desconocidos hasta ese momento. Entre éstos se encuentran la formulación de planes de recinto negociados con DAEM y Mineduc y acorde a la estimación de demanda de matrícula proyectada, el relato del uso cotidiano de los espacios educativos como petición de los arquitectos, la gestión de las orientaciones solicitadas por los arquitectos; la elaboración de reportes y rendiciones de cuenta para las sesiones de Mesa Técnica Comunal, la formulación de planes de mudanza y traslado, el registro de inventario y las diversas negociaciones para definir temas críticos del proyecto como postulación de fondos de equipamiento, selección de proveedores, requerimientos del diseño según Proyecto Educativo, entre otros. Fue un proceso intensivo en toma de decisiones sobre qué espacios construir acordes a sus proyectos educativos institucionales y cómo proyectar el uso pedagógico de estos espacios, por lo que los equipos directivos debieron organizarse, distribuirse responsabilidades y buscar internamente nuevos apoyos y liderazgos, que les permitieran mantener el sistema funcionando y responder adecuadamente a los requerimientos externos.

¿Qué hicieron estos establecimientos para que los resultados de aprendizaje no se vieran afectados ante una crisis de tamaña magnitud? La respuesta parece estar en que, en 
ambos casos, se mantuvo dentro del establecimiento el foco en lo pedagógico, y que las distintas acciones remediales que se llevaron a cabo en los establecimientos siempre tuvieron como objetivo final continuar el funcionamiento normal de los liceos y realizar las clases y planificaciones de la mejor manera posible. Ambos equipos tenían la convicción de que era necesario asegurar un buen proceso formativo para los estudiantes, independiente de la crisis de infraestructura.

$\mathrm{Si}$ bien es cierto que los directores debieron destinar mucho tiempo en el proceso de reconstrucción de sus liceos, un factor clave que ayudó a no perder el foco en lo pedagógico fue el liderazgo distribuido existente en ambas instituciones. En el caso de Liceo Mariano Latorre, el director se apoya en un extenso y proactivo equipo directivo, compuesto por 14 personas, entre los que hay docentes y asistentes de la educación, y aunque el liderazgo pedagógico queda instalado principalmente en la jefa de la Unidad Técnico-Pedagógica, durante los dos años en que el liceo se divide en dos sedes se tuvo que crear un rol transitorio de encargado de local. En ese sentido, el liceo ya contaba con una trayectoria donde los liderazgos estaban distribuidos. Sin embargo, la multiplicidad de personas en el mismo equipo a veces hacía que la toma de decisiones fuera más lenta y entorpecía los procesos. Fue por esto que durante el proceso el equipo se reestructuró, formando un equipo directivo y otro de gestión, con roles y tareas distintas.

El equipo directivo está compuesto por el Director, Jefa de UTP, Curriculista, Evaluadora,
Inspectora General, Orientadora, Coordinadores de las áreas TP y Escuela artística,
representante de los Profesores, Psicóloga, Psicopedagoga, Asistente Social y Jefe de
Finanzas. Si bien es un equipo altamente proactivo, es necesario revisar la organización de
este equipo multiprofesional, para establecer las funciones de un equipo de gestión y un
equipo directivo más operativo y eficiente. (Informe anual de consultoría institucional,
2011 )

El caso de Liceo Claudina Urrutia es más notorio, pues el equipo directivo distribuye el liderazgo entre los docentes, específicamente en los jefes de departamento, que son los encargados de continuar con la labor pedagógica del liceo.

El liderazgo directivo es democrático y ampliamente participativo, condición que ha sido demostrada en el proceso de reconstrucción. La comunidad educativa ha mantenido los altos niveles de adherencia al proyecto en esta segunda etapa. (Informe anual de consultoría institucional, 2012)

Los directores descansan en sus docentes y equipos directivos, donde el principal valor reside en la confianza que depositan en que son capaces de continuar sus labores de enseñanza sin la necesidad de que sean ellos mismos quienes estuvieran liderando dichos procesos.

La parte pedagógica puede que se nos tienda un poco a escapar... nos sentimos un poco lejos, por toda esta parte, pero tenemos confianza en que muchos profesores son personas muy comprometidas, entonces saben qué hacer en el aula cuando uno no está. (Directivo, LCU, oct -12 )

Se observaron durante el tiempo de crisis altos niveles de estrés en todos los estamentos, $\mathrm{y}$ algunos efectos negativos en el clima y la convivencia escolar, producto del hacinamiento y la falta de espacios de recreación dentro de los establecimientos, aunque también de algunas gestiones dentro del proceso de reconstrucción. Cabe destacar que también influyeron algunos factores externos al liceo, pues el terremoto de 2010 significó daños de gran magnitud en las comunidades. 
Muchos profesores y funcionarios también han tenido problemas después del terremoto, algunos siguen viviendo de allegados, entonces no es raro entender que esas personas se estresen. (Directivo, LCU, may-12)

El foco de acción de los directivos nunca estuvo puesto en la mejora del clima escolar o la reducción del estrés. El proceso planificado, de hecho, no tenía contemplado una línea de acción referido a este tema, a pesar de haber sido un tema de preocupación constante que fue canalizado hacia los equipos DAEM o de asesoría de Fundación Chile, ya que los actores se mostraban emocionalmente afectados por la crisis, lo que perjudicaba tanto el clima escolar como el desempeño laboral. Tan solo se realizaron algunas instancias que fueron generándose sobre la marcha como jornadas recreativas o actividades de relajación, las cuales ayudarían a paliar el estrés, pero dichas instancias fueron poco planificadas y sin continuidad, por lo que resultaron insuficientes para paliar algo esta deficiencia. Los niveles de estrés, sin embargo, fueron reducidos mediante las acciones que se verán a continuación.

Una de las medidas más importantes durante este proceso fue involucrar a la comunidad escolar en la solución de la crisis, es decir, hacerlos partícipes del proceso de reconstrucción. Esto incluyó una serie de acciones, como construir el diseño arquitectónico en base a sus opiniones y conocimientos -se realizó un estudio de necesidades y expectativas que consideró a toda la comunidad escolar-, informar constantemente de los avances e incluso organizar visitas guiadas a través de la obra cuando ya se estaba materializando. La amplia participación ayudaba a reducir la ansiedad y comprender que se está avanzando en salir de la crisis, que no se está estancado, sobre todo durante los 2 primeros años, etapa de diseño, en la que no existía un avance físico y visible, sino sólo maquetas y propuestas.

Los docentes están enfrentados a otra realidad que es estar en una sala pequeña, con muchos alumnos...Pero yo creo que la esperanza de lo que viene nos ayuda a disminuir los niveles de estrés. (Directivo, LCU, oct-12)

La importancia de la participación de la comunidad se manifestó sobre todo el año 2011, durante la etapa de anteproyecto y proyecto arquitectónico. Si bien en el Liceo Claudina Urrutia la participación se dio fácilmente, en el Mariano Latorre fue más complejo, pues en la cultura existente se destacaba más la acción y proactividad del equipo técnicodirectivo que la participación activa del resto de la comunidad. Así, en el contexto de las movilizaciones estudiantiles, en este liceo los alumnos adhirieron a las demandas nacionales, pero pusieron especial énfasis en la participación estudiantil, sobre todo en el mismo proceso de reconstrucción. En el Liceo Claudina Urrutia, en tanto, los alumnos no se movilizaron, en parte porque sentían que su liceo estaba avanzando.

[Hablando sobre un dibujo que expone] Quisimos destacar aquí -dice 'No más lucro'-el
tema de las movilizaciones de los estudiantes que también estuvo dentro de este proceso,
donde en algún momento los alumnos, dentro del petitorio nacional que existía, también
ellos de manera interna, particular, estuvieron demandando mayor participación, más
información, dentro del proceso de reconstrucción (...) Nos pareció que había sido un hito
dentro de este proceso. (Asistente de la Educación, LML, abr-14)

Ligado a la participación se encuentran dos elementos que van de la mano: la información que fluye a través de toda la comunidad escolar y la transparencia con la que se desarrolla el proceso para la solución de la crisis. Ambos elementos requieren de la existencia de una estrategia comunicacional sólida que considere a todos los actores escolares. 
Se observó cómo LCU tenía un estilo de entrega de información bastante ordenada, a través de una "Pauta Informativa" que se entregaba mensualmente a toda la comunidad, y asambleas donde el director personalmente explicaba los avances del proyecto. El liceo Mariano Latorre, en tanto, comenzó su proceso sin una estrategia clara de comunicación, lo que gatilló no sólo en desinformación, sino incluso en la difusión de rumores que no hicieron más que entorpecer el proceso y deteriorar el clima escolar.

Dentro de la construcción hay considerados 4 laboratorios que están considerados para esto, y el ministerio no va construir para esta especialidad, no hay espacio. El problema es que después tú te enteras, que esos espacios se los prometieron a otras áreas. Esas cosas, entonces uno no haya a quién creerle, hay información que se está ocultando. (Docente, LML, jul11)

Uno de los principales hallazgos de este estudio muestra como en el Liceo Claudina Urrutia las fuentes de información estaban centralizadas en el director desde el principio, y que la bajada a los alumnos se apoya además en los profesores jefes, mientras que en el Liceo Mariano Latorre existía una multiplicidad de fuentes de información que deterioraban su calidad. Lo mismo se relacionaba finalmente con los niveles de información percibidos por los mismos actores de los liceos. Los gráficos 3 y 4 ilustran esto a través de la opinión de los alumnos al principio del proceso, mientras que los gráficos 5 y 6 ilustran cómo en un plazo de 18 meses en el liceo Mariano Latorre logran centralizar, en la medida de lo posible, la información hacia los alumnos a través de profesores y medios oficiales como las redes sociales o el boletín escolar, y aumentan los niveles de información de forma significativa, aunque condicionados por una cultura escolar previa que no consideraba la necesidad de esta centralización, lo que hace que los aumentos no lleguen a los niveles de centralización e información del Liceo Claudina Urrutia.

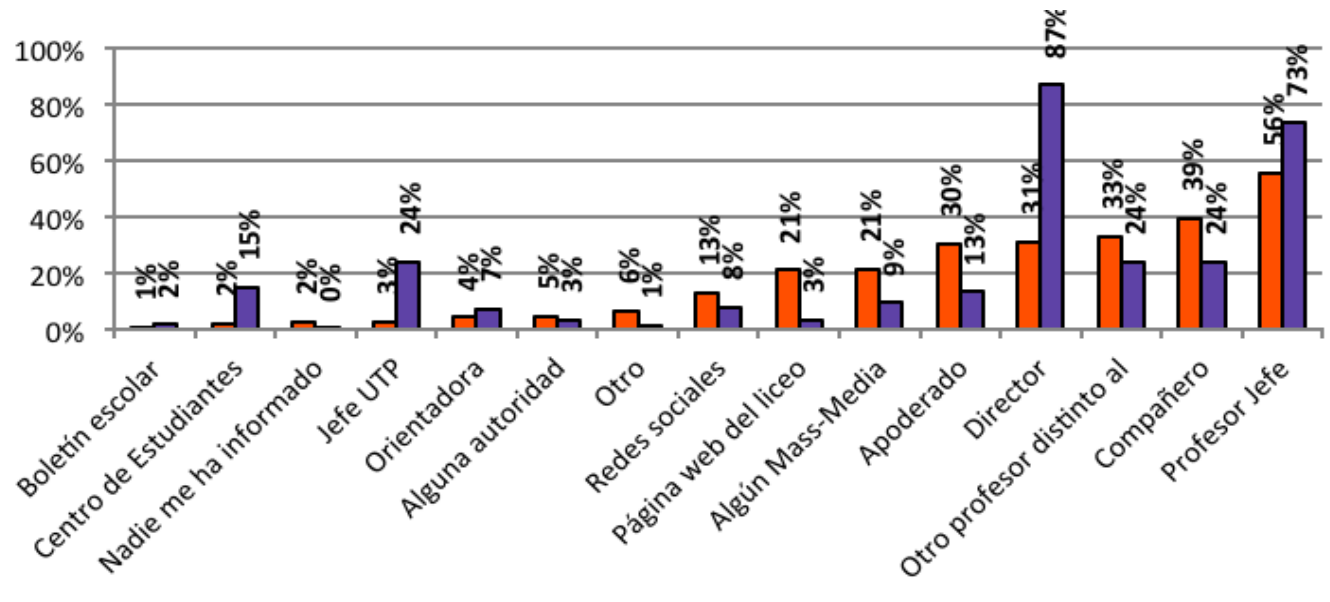

$\square \mathrm{LML} \quad \square \mathrm{LCU}$

Gráfico 3. Fuentes de información de estudiantes sobre proceso de reconstrucción según Liceo

Fuente: Encuesta a alumnos aplicada en abril de 2011.

El sólo hecho de informar y conocer el proyecto crea un sentido de participación, de estar presentes en el proceso. Por lo mismo, luego de conocidos estos datos el liceo intentó realizar algunas acciones para mejorar sus flujos de comunicación. En principio se intentaron imitar las estrategias que aplicaba el Liceo Claudina Urrutia, pero no 
tuvieron el éxito esperado, pues no estaba dentro de la cultura escolar el leer boletines mensuales, mientras que en el otro liceo se daba esta práctica desde hacía más de 15 años, y se hacía difícil concretar asambleas con el liceo dividido. Fue necesario tomar estrategias más complejas pero, a la vez, más acordes con la cultura escolar existente, utilizando medios como la radio del liceo y el apoyo de los profesores jefes en la difusión.

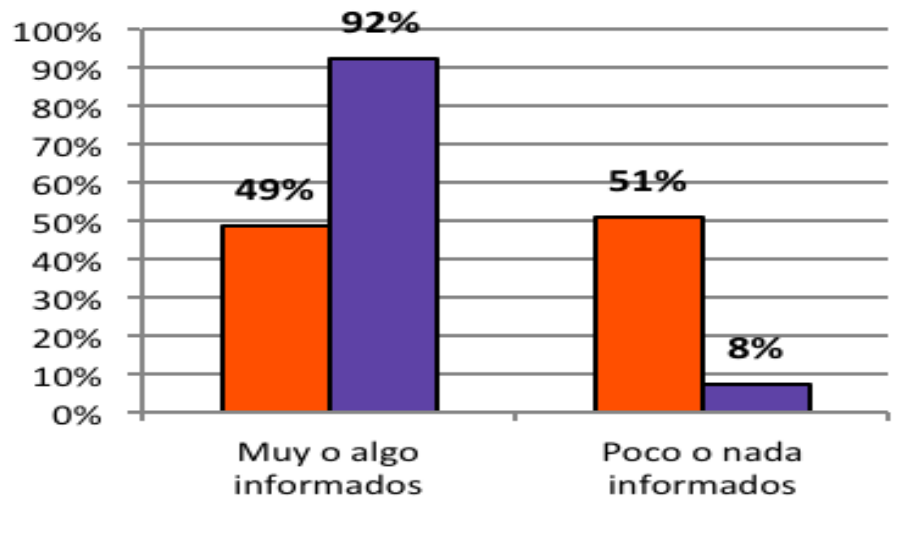

$\square \mathrm{LML} \square \mathrm{LCU}$

$N=634$

Gráfico 4. Nivel de información de estudiantes sobre procesos de reconstrucción según Liceo

Fuente: Encuesta a alumnos aplicada en abril de 2011.

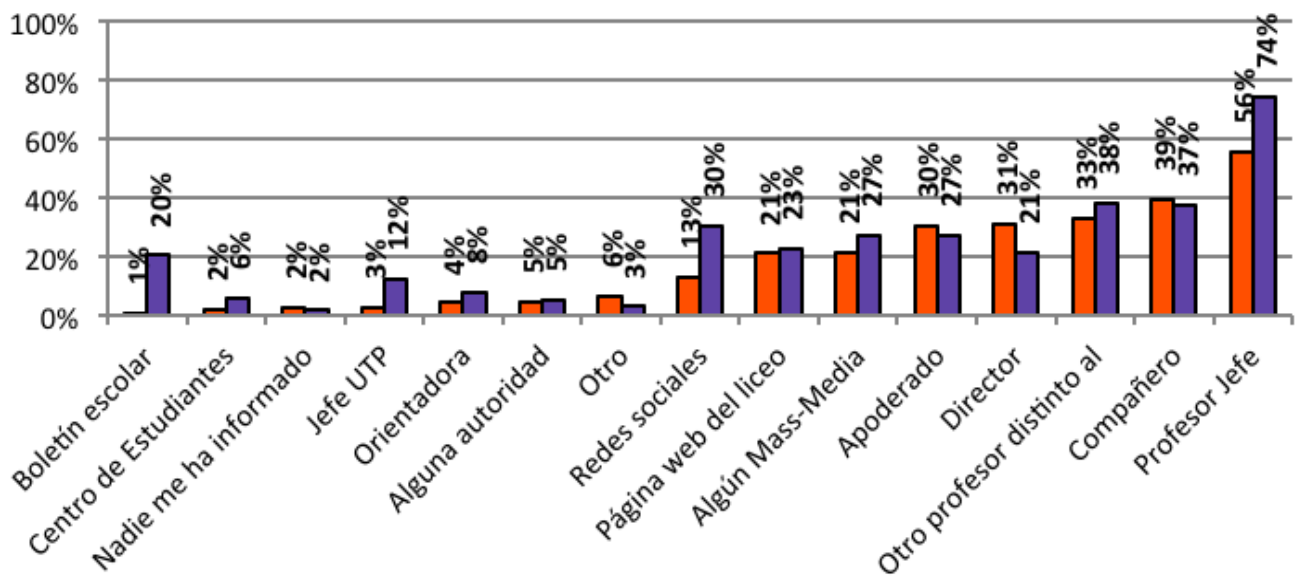

$\square_{a b r-11} \mathbf{a}_{\mathrm{oct}}-12$

Gráfico 5. Fuentes de información de estudiantes LML sobre reconstrucción según fecha Fuente: Encuestas a alumnos aplicadas en abril de 2011 y octubre de 2012.

Un último punto que vale la pena mencionar es que durante todo el proceso destacó dentro de ambos establecimientos que desde un principio mostraron permeabilidad ante la asesoría otorgada por Fundación Chile. El "dejarse ayudar” pareció haber jugado un rol clave durante todo el proceso, y desde la misma consultoría se percibía que los liceos se mostraron tal cual, exponiendo sus virtudes y sus defectos con el objetivo de guiar el proceso de la mejor manera posible. Si bien la catástrofe pudo haber generado una reacción contraria, generando esfuerzos y gastos de energía innecesarios para parecer 
firmes ante la adversidad, la actitud de transparencia permitió que la consultoría se desarrollara de mejor manera.

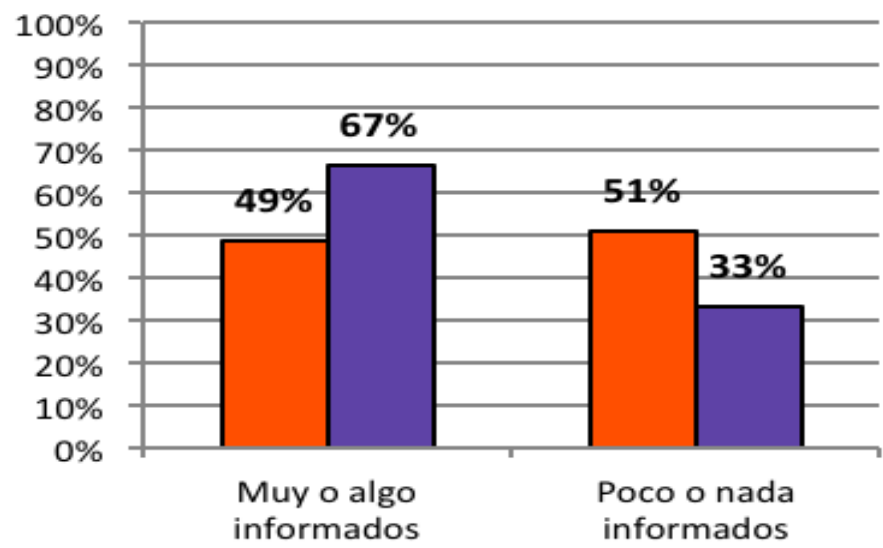

口abr-11 $\square$ oct-12

$$
\mathrm{N}=634
$$

Gráfico 6. Nivel de información de estudiantes LML sobre proceso de reconstrucción según fecha

Fuente: Encuestas a alumnos aplicadas en abril de 2011 y octubre de 2012.

\section{Conclusiones y Discusión}

Varios aprendizajes surgen de las experiencias revisadas. ¿Cómo mantener los resultados académicos mientras se debe gestionar la resolución de una gran crisis? Los resultados arrojan que las prácticas debieran desenvolverse en tres grandes líneas: La mantención del foco en lo pedagógico; la distribución ordenada de liderazgos y participación de la comunidad escolar; y la existencia de canales de comunicación sólidos.

La primera línea es fundamental. A pesar de que la crisis apunte en el sentido contrario, y la tendencia del equipo directivo pueda ser el preocuparse de solucionar una crisis, lo importante es que el establecimiento vuelva a su funcionamiento normal en lo que refiere a enseñanza y aprendizaje, independiente de otros elementos que puedan afectar este proceso. Esto va en la línea de lo que dicen Elaqua y sus colegas (2010), cuando rescatan la primordialidad de reestablecer tanto las condiciones físicas del establecimiento como las prácticas pedagógicas y las metas de mejora propuestas lo antes posible. Mantener este foco es complejo ante la tentación de atender los problemas más urgentes y visibles, que tampoco deben descuidarse, y es por esto que las otras dos líneas resultan un apoyo para resguardar ésta.

Para la segunda línea, la distribución ordenada de liderazgos, el liderazgo distribuido sería la clave, donde la confianza en los docentes y los equipos técnicos se combinan con la legitimidad de un equipo directivo sólido y con trayectoria. La distribución de responsabilidades fue fundamental para que el equipo directivo no se viese colapsado frente a la enorme cantidad de nuevas tareas que puede traer una crisis, muchas de estas ajenas al trabajo pedagógico y rutinario (como gestión logística, entre otros). Esto estaría muy acorde con lo que plantean Robinson, Hohepa y Lloyd (2009), sobre que un liderazgo sólido es fundamental para mantener el foco en los resultados educativos, elemento esencial durante una crisis. En la misma línea, toma fuerza el liderazgo 
distribuido, ya que como señala Harris (2012), potencia que los docentes se apropien de las prácticas a realizar, lo cual, según lo observado, fue fundamental en los liceos para que el foco en lo pedagógico se mantuviese. En otras palabras, fue necesario que los docentes tomaran un papel más protagónico frente a las nuevas circunstancias, y se hicieran cargo de los pasos a realizar, para así poder mantener el foco educativo. Sin embargo, se observó en los casos estudiados que esta distribución de funciones no siempre era beneficiosa, pues que hubiese muchas personas tomando decisiones podía complejizar ciertos procesos, haciéndose necesario que dicha distribución fuera planificada, otorgando a cada líder un rol claro. Esto es así, ya que en tiempos de crisis las decisiones deben ser más ágiles y oportunas, y en muchos casos pueden existir respuestas anticipadas a las problemáticas emergentes, haciéndose fundamental optimizar los tiempos y recursos. Para el liderazgo distribuido no sólo es importante que se distribuyan las responsabilidades pedagógicas para afrontar dificultades, sino que también se promueva la participación de toda la comunidad educativa en la toma de decisiones (Harris, 2002; García, 2010; Murillo, 2006). En los liceos se confirmó que era fundamental incluir a la comunidad educativa, no sólo docentes y directivos, en los procesos de reconstrucción de las instituciones, pues permitía reducir los niveles de ansiedad de los integrantes de la comunidad escolar al tiempo de mejorar los compromisos y la identidad con la institución.

Apoyando esta segunda línea de acción, se observó que para lograr un mayor liderazgo y compromiso existe la necesidad de que haya una estrategia de comunicación sólida y espacios de participación organizados con una sólida conducción y propósito que permitan que ésta pueda ser sistémica y acorde a las necesidades del establecimiento. Esto estaría fuertemente en concordancia con lo presentado en la literatura, donde Transberg (2012) resalta la importancia de la información sobre los pasos a seguir y le rendición en cuentas para entregar una mayor seguridad a los actores frente a un conflicto o una crisis. Pero además, según indica la experiencia, parece fundamental que la comunicación interna sea fluida y a la vez ordenada, cuidando que los canales de información estén unificados para evitar la mala información y el desarrollo de una cultura del rumor que pueden ser dañinos para la institución. Como señalan Sotomayor Y Sánchez (2010), la desinformación entre los programas o proyectos asesores o que guían la reconstrucción, y la comunidad educativa puede generar resistencia por parte de la escuela, siendo fundamental asegurar una buena relación entre ambos, flexibilizando horarios, siendo empáticos, cumpliendo los compromisos, y enfocándose en una meta común, entre otros. Es así que en esta misma línea Fundación Chile, en el caso de los liceos estudiados, apoyó en generar una buena comunicación dentro de la escuela, preocupándose de mantener un eje común y conocido con la escuela en todo momento.

Un aspecto que llama la atención en los casos observados es que no se puso foco en reducir los niveles de estrés directamente, a pesar de la importancia de velar por el estado anímico de los docentes y el resto de la comunidad escolar, como señala Leithwood (2009). Sin embargo, si bien no existió un trabajo sistemático con el objetivo directo de reducir el estrés, se observó que la preocupación constante por mantener un proceso participativo y con flujos de información sólidos incidían en los niveles de ansiedad y estrés de la comunidad escolar y mantenían estable la calidad del clima escolar.

Cualquier estrategia que se tome en las tres líneas debe tener en cuenta el contexto en las que se enmarcan. Se debe recordar que las estrategias que fueron efectivas en ambos 
liceos respondieron a prácticas que se desarrollaban con anterioridad en cada uno de ellos. De esto surge como un nuevo aprendizaje que cualquier estrategia dentro de una crisis debe adaptarse a la cultura escolar del establecimiento, pues la emergencia y contingencias propias de una crisis no dan espacio para que los actores realicen un esfuerzo adicional para adecuarse a estrategias desconocidas. De hecho, en un informe del Programa de Naciones Unidas para el Desarrollo (PNUD) sobre la recuperación y reconstrucción post-terremoto (2012) se señala la importancia de diseñar una estrategia de afrontamiento de la crisis adecuada a la realidad de la zona y acorde con su cultura, indicando que frente a procesos de reconstrucción es importante respetar las costumbres de la comunidad y el medio natural, considerando tanto devolver la funcionalidad a la institución como recuperar la seguridad y entregar cobijo a la población afectada, mediante la mantención de su identidad en la obra. Así mismo, Elmore (2010) menciona que para que cualquier política educativa sea exitosa debe ser acorde a las prácticas pedagógicas y la cultura del establecimiento, dándole relevancia a su contexto particular, de manera de poder desarrollar medidas acordes a su realidad, generando cohesión y una cultura pedagógica común como establecimiento. De ahí que además se vuelve importante que haya una actitud de transparencia y apertura ante las asesorías, que permita que las estrategias propuestas se adapten a la cultura escolar. En la misma línea, es necesario que los establecimientos educativos cuenten con una estructura organizacional y una cultura escolar sólida y preparada que facilite afrontar una crisis antes de que suceda, de modo que ésta no exija más trabajo de los que por naturaleza pueda demandar.

El presente estudio abre las puertas para investigar temas de cultura organizacional dentro de las escuelas como participación, comunicación interna y distribución de responsabilidades, no sólo en contextos de crisis, sino como elementos fundamentales dentro de las culturas escolares. No se puede olvidar que, como estudio de caso, este artículo sólo pretende dar algunas claves, pero será necesario profundizar en cada una de las líneas descubiertas para poder generar estrategias que se adapten a cualquier contexto escolar.

\section{Referencias}

Bellei, C., Raczynski, D. y Osses, A. (2010). ¿Qué hemos aprendido sobre programas de asistencia técnica educativa? Análisis colectivo de los estudios de caso. En C. Bellei (Ed.), Asistencia técnica educativa en chile: ¿aporte al mejoramiento escolar? (pp. 34-76). Santiago: Ocho Libros.

Elacqua, G., Santos, H. y Salazar, F. (2010). Terremoto 27/2: oportunidad para mejorar las escuelas en Chile. Santiago: CPCE Centro de Política Comparada en Educación (CPCE).

Elmore, R. (2010). Mejorando la escuela desde la sala de clases. Santiago: Área de Estudio de Fundación Chile.

Fried Schnitman, D. (2011). Afrontamiento generativo de crisis y conflictos en organizaciones. Persona, 14, 11-44.

García, I. (2010). Liderazgo distribuido, una visión innovadora de la dirección escolar: una perspectiva teórica. Omnia, 16(3), 19-36.

Harris, A. (2012). Liderazgo y desarrollo de capacidades en la escuela. Santiago: Centro de Innovación en Educación. 
Horn, A. y Marfán, J. (2010). Relación entre liderazgo educativo y desempeño escolar. Revisión de la investigación en Chile. Psicoperspectivas, 9(2), 82-104. doi: 10.5027/psicoperspectivasvol9-issue2-fulltext-116

INDH, I.N. (2012). $27 \mathrm{~F}$. Estudio sobre la reconstrucción post terremoto desde una perspectiva de derechos humanos. Santiago: INDH.

Leithwood, K. (2009). La dimensión emocional del mejoramiento escolar: una perspectiva desde el liderazgo. En K. Leithwood (Coord.), ¿Cómo liderar nuestras escuelas? aportes desde la investigación (pp. 175-200). Santiago: Área de Educación Fundación Chile.

Leithwood, K. y Stager, M. (2009). Competencias de los directores en la solución de problemas. En K. Leithwood (Coord.), ¿Cómo liderar nuestras escuelas? aportes desde la investigación (pp. 135-173). Santiago: Área de Educación de Fundación Chile.

Murillo, F.J. (2006). Una dirección escolar para el cambio: del liderazgo transformacional al liderazgo distribuido. REICE. Revista Iberoamericana sobre Calidad, Eficacia y Cambio en Educación, 4(4), 11-24.

MINEDUC. (2013). La reconstrucción en Educación. Una mirada a los procesos y desafíos de la reconstrucción de la infraestructra escolar dañada en el terremoto y maremoto del $27 / F$. Santiago: MINEDUC.

PNUD. (2012). Recuperación y reconstrucción post desastre. Experiencias y herramientas de aplicación a nivel regional y local. Santiago: PNUD.

Reeves, M. (2012). Efectos del liderazgo directivo en escuelas con altos niveles de vulnerabilidad social. En J. Weinstein y G. Muñoz (Eds.), ¿QQué sabemos sobre los directores de escuela en Chile? (pp. 307-324). Santiago: Centro de Innovación en Fundación Chile y CEPPE.

Robinson, V., Hohepa, M. y Lloyd, C. (2009). School leadership and student outcomes: identifying what works and why. Best evidence synthesis iteration (BES). Wellington: Ministerio de Educación.

Sotomayor, C. y Sánchez, M. (2010). Programa interactivo para la educación básica fundación educacional arauco. En C. Bellei (Ed.), Asistencia técnico educativa en Chile: ¿Aporte al mejoramiento escolar? (pp. 149-182). Santiago: Ocho Libros.

Transberg, M. (2012). La cooperación internacional en la reconstrucción de Haití: un acercamiento desde la perspectiva de la eficacia de la ayuda. Salamanca: Instituto de Iberoamérica.

Universidad Diego Portales. (2010). Informe anual sobre derechos humanos. Santiago: Centro de Derechos Humanos.

Valenzuela, P. y Horn, A. (2012). Influencia del liderazgo directivo en los resultados de los estudiantes. En J. Weinstein y G. Muñoz (Eds.), ¿Qué sabemos sobre los directivos de la escuela en Chile? (pp. 325-348). Santiago: Centro de Innovación en Fundación Chile y CEPPE. 